\title{
A NEW RESULT FOR THE POROUS MEDIUM EQUATION DERIVED FROM THE RICCI FLOW
}

\author{
LANG-FANG WU
}

\begin{abstract}
Given $\mathbb{R}^{2}$, with a "good" complete metric, we show that the unique solution of the Ricci flow approaches a soliton at time infinity. Solitons are solutions of the Ricci flow, which move only by diffeomorphism. The Ricci flow on $\mathbb{R}^{2}$ is the limiting case of the porous medium equation when $m$ is zero. The results in the Ricci flow may therefore be interpreted as sufficient conditions on the initial data, which guarantee that the corresponding unique solution for the porous medium equation on the entire plane asymptotically behaves like a "soliton-solution".
\end{abstract}

On $\mathbb{R}^{2}$, any metric can be expressed as $d s^{2}=e^{u}\left(d x^{2}+d y^{2}\right)$, where $\{x, y\}$ are rectangular coordinates on $\mathbb{R}^{2}$. Let $R$ be the scalar curvature, then the so-called Ricci flow on $\mathbb{R}^{2}$ is

$$
\frac{\partial}{\partial t} d s^{2}=-R d s^{2}
$$

which may also be expressed as

$$
\frac{\partial}{\partial t} u=e^{-u} \bar{\Delta} u, \quad \text { where } \bar{\Delta}=\partial_{x}^{2}+\partial_{y}^{2}
$$

The porous medium equation is defined as

$$
\frac{\partial}{\partial \tau} v=\bar{\Delta} v^{m}
$$

where $0<m<\infty$, and $v$ is a function on $\mathbb{R}^{2}$. If we let $\tau=t / m$, then $\left({ }^{*} 2\right)$ can be expressed as

$$
\frac{\partial}{\partial t} v=\frac{\bar{\Delta}\left(v^{m}-1\right)}{m}
$$

The limiting case of the porous medium equation as $m \rightarrow 0$ [PME $(m=0)]$ is therefore

$$
\frac{\partial}{\partial t} v=\bar{\Delta} \ln v
$$

In dimension 1 , the PME $(m=0)$ with initial data $v \notin L^{1}$ has been studied by [ERV, $\mathrm{H} 1, \mathrm{H} 2, \mathrm{~V}]$.

We are grateful to Sigurd B. Angenent for pointing out the following observation reflected in [W2, Appendix].

Received by the editors September 24, 1991 and, in revised form, on November 13, 1991 and May 1, 1992. Presented on July 2 at the 1992 Regional Geometry Institute at Utah.

1991 Mathematics Subject Classification. Primary 58D25 35K05. 
Proposition [Angenent]. On $\mathbb{R}^{2}$, if we let $v=e^{u}$, then the limiting case of the porous medium equation as $m \rightarrow 0 \quad\left({ }^{*} 3\right)$ is equivalent to the Ricci flow $\left({ }^{*} 1\right)$.

On a complete $\left(\mathbb{R}^{2}, d s^{2}\right)$ we may define:

(a) the circumference at infinity to be

$$
C_{\infty}\left(d s^{2}\right)=\sup _{K} \inf _{D^{2}}\left\{L\left(\partial D^{2}\right) \mid \forall \text { compact set } K \subset \mathbb{R}^{2}, \forall \text { open set } D^{2} \supset K\right\}
$$

where $L\left(\partial D^{2}\right)$ is the length of $\partial D^{2}$ with respect to the metric $d s^{2}$;

(b) the aperture to be

$$
A\left(d s^{2}\right)=\frac{1}{2 \pi} \lim _{r \rightarrow \infty} \frac{L\left(\partial B_{r}\right)}{r},
$$

where $B_{r}$ is a geodesic ball at any given point on $\mathbb{R}^{2}$ with radius $r$.

A gradient soliton is a solution of the Ricci flow, which moves only by diffeomorphism and there exists a function $f$ such that $\frac{\partial}{\partial t} g_{i j}=L_{\nabla f} g_{i j}$, where $L_{\nabla f}$ is the Lie derivative in the direction of the gradient $\mathrm{f}$. There are two types of gradient solitons on $\mathbb{R}^{2}$. Namely, the flat soliton $\left(C_{\infty}=\infty, A=1\right)$ and the cigar soliton $\left(C_{\infty}<\infty, A=0\right)$. The flat soliton is the standard flat metric on $\mathbb{R}^{2}$. The cigar soliton is a metric, which can be expressed as $d s^{2}=\left(d u^{2}+d v^{2}\right) /\left(1+u^{2}+v^{2}\right)$, where $\{u, v\}$ are rectangular coordinates on $\mathbb{R}^{2}$. The cigar solitons are the so-called Barenblatt solutions in the field of the porous medium equations.

The Ricci flow and the soliton phenomenon gave a new proof for the uniformization theorem on compact surfaces and orbifolds without boundary ([C, $\mathrm{Ha} 1, \mathrm{~W} 2, \mathrm{CW}])$. Understanding the solitons may provide insights toward studying the Ricci flow on higher-dimensional Kähler manifolds. For an announcement of related work, see [Shi]. As a step towards further studying the soliton phenomenon on higher-dimensional Kähler manifolds, Richard Hamilton raised the following question:

Question. On what manifolds do solutions to the Ricci flow asymptotically approach nontrivial solitons?

One of the simplest spaces is $\mathbb{R}^{2}$ with complete metric. We say that the Ricci flow on $\mathbb{R}^{2}$ has weak modified convergence at time infinity if there exists a 1-parameter family of diffeomorphisms $\left\{\phi_{t}\right\}_{t \in[0, \infty)}$ on $\mathbb{R}^{2}$ such that for any sequence of times going to infinity there is a subsequence of times $\left\{t_{j}\right\}_{j=0}^{\infty}$ and the modified metric $d s^{2}\left(\phi_{t_{j}}(\cdot), t_{j}\right)$ converges uniformly on every compact set as $j \rightarrow \infty$. We will now state our results concerning the Ricci flow.

Main Theorem (Ricci flow [W2]). Given a complete $\left(\mathbb{R}^{2}, d s^{2}(0)\right)$ with $|R| \leq C$ and $|D u| \leq C$ at $t=0$, the Ricci flow has weak modified convergence at time infinity to a limiting metric. In the case when $R>0$ at $t=0$, the limiting metric is a cigar soliton if $C_{\infty}\left(d s^{2}(0)\right)<\infty$, or a flat metric if $A\left(d s^{2}(0)\right)>0$.

In the process of proving the main theorem we have the following lemmas. Let $R$ denote the scalar curvature and $R_{-}=\max \{-R, 0\}$.

Lemma 1 (Ricci flow) (Long time existence). Given a complete $\left(\mathbb{R}^{2}, d s^{2}\right)$ with $|R| \leq C$ and $|D u| \leq C$ at $t=0$, under the Ricci flow $\left({ }^{*} 1\right)$, the solution of the flow exists for infinite time. 
Lemma 2 (Ricci flow). Given a complete $\left(\mathbb{R}^{2}, d s^{2}\right)$ with $|R| \leq C, \int_{\mathbb{R}^{2}} R \_d \mu<$ $\infty,|D u| \leq C$, and $C_{\infty}>0$ at $t=0$, under the Ricci flow $\left({ }^{*} 1\right)$, we have the following:

1. (Uniqueness) The solution of the flow is unique.

2. (Geometric Properties) $C_{\infty}, A\left(g_{i j}\right)$, and $\int_{\mathbb{R}^{2}} R d \mu<\infty$ are constants under the flow.

Lemma 3 (Ricci flow). Given a complete $\left(\mathbb{R}^{2}, d s^{2}\right)$ with $0<R \leq C$ and $|D u| \leq C$ at $t=0$. Then, under the Ricci flow $\left({ }^{*} 1\right), \lim _{t \rightarrow \infty} e^{u(x, y, t)}$ converges uniformly on every compact set and $\lim _{t \rightarrow \infty} e^{u(x, y, t)}$ is either identically zero or positive everywhere. If $\lim _{t \rightarrow \infty} e^{u(x, y, t)}>0$ then $\lim _{t \rightarrow \infty} e^{u(x, y, t)}\left(d x^{2}+d y^{2}\right)$ induces a metric on $\mathbb{R}^{2}$ with curvature identically zero.

Nevertheless, it is possible to choose a 1-parameter family of diffeomorphisms to get weak modified convergence. To see why modifying the solution by diffeomorphism is needed, we will illustrate the following example.

Example 2.2. Given a cigar soliton (or one of the Barenblatt solutions) $d s^{2}(0)=$ $\left(d x^{2}+d y^{2}\right) /\left(1+x^{2}+y^{2}\right)$ on $\mathbb{R}^{2}$, it is easy to compute that the solution of the Ricci flow with initial data $d s^{2}(0)$ is $d s^{2}(t)=\left(d x^{2}+d y^{2}\right) /\left(e^{4 t}+x^{2}+y^{2}\right)$. Then $e^{u(x, y, t)}=1 /\left(e^{4 t}+x^{2}+y^{2}\right)$ goes to zero as time approaches infinity; therefore, we cannot claim that $\lim _{t \rightarrow \infty} e^{u(x, y, t)}$ yields a metric on $\mathbb{R}^{2}$.

Nevertheless, if we let diffeomorphism $\phi_{t}(A, B)=\left(e^{2 t} A, e^{2 t} B\right)=(x, y)$, then

$$
d s^{2}(x, y, t)=d s^{2}\left(\phi_{t}(A, B), t\right)=\frac{d A^{2}+d B^{2}}{1+A^{2}+B^{2}} .
$$

Let $\widehat{d s^{2}}(A, B, t)=d s^{2}\left(\phi_{t}(A, B), t\right)$ and $e^{\widehat{u}}=1 /\left(1+A^{2}+B^{2}\right)$. Then $e^{\widehat{u}}$ is stationary in time.

Note that the Ricci flow on other complete noncompact surfaces is also discussed in [W2].

Now we will list the corresponding relations between the function $v$ and the geometric properties. If $\{r, \theta\}$ are polar coordinates on $\mathbb{R}^{2}$, then $d s^{2}=$ $v\left(d r^{2}+r^{2} d \theta^{2}\right)$ and

$$
\begin{aligned}
\left(\mathbb{R}^{2}, d s^{2}\right) \text { is complete } \Longrightarrow v>0 \text { and } \int_{r=0}^{\infty} v^{1 / 2}(\theta, r) d r & =\infty \\
& \forall 0 \leq \theta \leq 2 \pi,
\end{aligned}
$$

$$
\begin{gathered}
|R| \leq C \Longleftrightarrow\left|\frac{\bar{\Delta} \ln v}{v}\right| \leq C, \\
0<R \leq C \Longleftrightarrow 0<-\frac{\bar{\Delta} \ln v}{v} \leq C, \\
|D u| \leq C \Longleftrightarrow\left|\left(v_{x}^{2}+v_{y}^{2}\right) / v^{3}\right| \leq C^{2} ; \\
\int R_{-} d \mu \leq C \Longleftrightarrow \int \max \{-\bar{\Delta} \ln v, 0\} d x d y \leq C,
\end{gathered}
$$




$$
\begin{gathered}
C_{\infty}=\lim _{r \rightarrow \infty} \int r v^{1 / 2} d \theta>0 \Longrightarrow v \notin L^{1}, \\
A\left(d s^{2}\right)=\lim _{r \rightarrow \infty} \frac{\int r v^{1 / 2} d \theta}{\int v^{1 / 2} d r}>0 .
\end{gathered}
$$

The relations $\left({ }^{*} 5\right),(* 6)$, and $(* 8)$ follow from

$$
\begin{gathered}
R=-e^{-u} \bar{\Delta} u=-\frac{\bar{\Delta} \ln v}{v}, \\
u=\ln v \quad \text { and }|D u|^{2}=\frac{1}{v} \frac{\left.<v_{x}, v_{y}\right\rangle}{v} \cdot \frac{\left\langle v_{x}, v_{y}\right\rangle}{v}, \\
\int v d x d y=\int e^{u} d x d y=\infty
\end{gathered}
$$

We say that the PME $(m=0)$ on $\mathbb{R}^{2}$ has weak modified convergence at time infinity if there exists a 1-parameter family of reparametrizations $\left\{\phi_{t}\right\}_{t \in[0, \infty)}$ on $\mathbb{R}^{2}$ such that for any sequence of times going to infinity there is a subsequence of times $\left\{t_{j}\right\}_{j=0}^{\infty}$ and the modified solution $v\left(\phi_{t_{j}}(\cdot), t_{j}\right)$ converges uniformly to a positive function on every compact set as $j \rightarrow \infty$. Then we have

Main Theorem* [PME $(m=0)]$. On $\mathbb{R}^{2}$, let the positive function $v$ satisfy $\left({ }^{*} 4\right),\left({ }^{*} 5\right)$, and $\left({ }^{*} 6\right)$ at $t=0$. Then, under the PME $(m=0)$, the solution $v(x, t)$ of PME $(m=0)$ with $v(x, 0)=v(x)$ has weak modified convergence at time infinity to a limiting positive function $v_{\infty}$ satisfying $\left({ }^{*} 4\right)$. In the case when $\left(* 5^{\prime}\right)$ also holds at $t=0, v_{\infty}$ is one of the Barenblatt solutions if $C_{\infty}(v(\cdot, 0))<$ $\infty$, or a constant if $A(v(\cdot, 0))>0$.

Lemma *1 [PME $(m=0)$ ] (Long time existence). On $\mathbb{R}^{2}$, if the positive function $v$ satisfies $\left({ }^{*} 4\right),\left({ }^{*} 5\right)$, and $\left({ }^{*} 6\right)$ at $t=0$, then, under the PME $(m=0)$, the solution $v(x, t)$ of PME $(m=0)$ with $v(x, 0)=v(x)$ exists for infinite time.

Lemma $* 2(\operatorname{PME}(m=0))$. On $\mathbb{R}^{2}$, if the positive function $v$ satisfies $\left({ }^{*} 4\right),\left({ }^{*} 5\right)$, $\left({ }^{*} 6\right),\left({ }^{*} 7\right)$, and $\left({ }^{*} 8\right)$ at $t=0$, then, under the PME $(m=0)$, we have the following:

1. (Uniqueness) The solution of the flow is unique.

2. (Geometric Properties) $C_{\infty}, A\left(d s^{2}\right)$, and $\int_{\mathbb{R}^{2}} R d \mu<\infty$ are constants under the flow.

Lemma *3 (PME $(m=0))$. On $\mathbb{R}^{2}$, if the positive function $v$ satisfies $\left({ }^{*} 4\right)$, $\left({ }^{*} 5^{\prime}\right)$, and $\left({ }^{*} 6\right)$ at $t=0$, then, under the PME $(m=0), \lim _{t \rightarrow \infty} v(x, y, t)$ converges uniformly on every compact set and $\lim _{t \rightarrow \infty} v(x, y, t)$ is either identically zero or positive everywhere. If $\lim _{t \rightarrow \infty} v(x, y, t)>0$ then $\lim _{t \rightarrow \infty} v(x, y, t)\left(d x^{2}+d y^{2}\right)$ induces a metric on $\mathbb{R}^{2}$ with curvature identically zero, in particular, $\lim _{t \rightarrow \infty} v(x, y, t)$ is a constant.

Note that there is still a large class of Riemannian structures with $C_{\infty}=\infty$ and $A=0$, which our method fails to classify the limit.

Sketch of the proof. The evolution equation of $h=R+|D u|^{2}$ provides the infinite time existence and uniform bounds for $|D u|,\left|D^{k} u\right|, R$, and $\left|D^{k} R\right|$ 
for all $k \geq 1$ after a short time. Finite total curvature and $C_{\infty}>0$ imply that the curvature decays to zero at distance infinity. This yields that $C_{\infty}, A\left(d s^{2}\right)$, and $\int R d \mu$ are preserved under the flow. Furthermore, the solution of the flow is unique and the injectivity radius $i(M)$ decays at most exponentially.

The positivity of the curvature of an initial metric provides pointwise convergence of the function $e^{u}$ at time infinity. The uniform bounds on $\left|D^{m} u\right|$ imply $\lim _{t \rightarrow \infty} e^{u}$ is a smooth function and is either identically zero or positive everywhere. In the case, when $\lim _{t \rightarrow \infty} e^{u}>0$, the limiting solution is a flat metric.

We also may choose a 1-parameter family of diffeomorphisms $\phi_{t}: \mathbb{R}^{2} \rightarrow \mathbb{R}^{2}$ such that there exists a sequence of times $\left\{t_{j}\right\}_{j=0}^{\infty}$ and $\lim _{t \rightarrow \infty} d s^{2}\left(\phi_{t_{j}}(\cdot), t_{j}\right)$ converges uniformly on every compact set. If $R>0$ at $t=0$ and $C_{\infty}\left(d s^{2}(0)\right)<$ $\infty$, then some integral bound classifies $\lim _{t \rightarrow \infty} d s^{2}\left(\phi_{t_{j}}(\cdot), t_{j}\right)$ as a cigar soliton with circumference no bigger than $C_{\infty}\left(d s^{2}(0)\right)<\infty$. If $R>0$ at $t=0$ and $A\left(d s^{2}(0)\right)>0$, then the Harnack's inequality classifies $\lim _{t \rightarrow \infty} d s^{2}\left(\phi_{t_{j}}(\cdot), t_{j}\right)$ as a flat metric.

\section{REFERENCES}

[A] D. G. Aronson, The porous medium equations, Some Problems in Nonlinear Diffusion (A. Fasano and M. Primicerio, eds.), Lecture Notes in Maths., vol. 1224, Springer, New York, 1986.

[CW] B. Chow and L. Wu, The Ricci flow on compact 2-orbifolds with curvature negative somewhere, Comm. Pure and Appl. Math., vol. XLIV, Wiley, New York, 1991, pp. 275-286.

[ERV] J. R. Esteban, A. Rodriguez, and J. L. Vazquez, A nonlinear heat equation with singular diffusivity, Arch. Rational Mech. Analysis 103 (1988), 985-1039.

[Ha1] R. Hamilton, The Ricci flow on surfaces, Contemp. Math., vol. 71, Amer. Math. Soc., Providence, RI, 1988, pp. 237-262.

[Ha2] - Notes on Harnack's inequality, preprint.

[H1] M. A. Herrero, A limiting case in nonlinear diffusion, Nonlinear Anal. 13 (1989), 611-628.

[H2] Singular diffusion on the line (to appear).

[Shi] W. X. Shi, Complete noncompact Kähler manifolds with positive holomorphic bisectional curvature, Bull. Amer. Math. Soc. (N.S.) 23 (1990), 437-440.

[V] J. L. Valazquez, Two nonlinear diffusion equations with finite speed of propagation, Proceedings of the conference in honor of Jack Hale on the occasion of his 60th birthday, preprint.

[W1] L. Wu, The Ricci flow on 2-orbifolds with positive curvature, J. Differential Geom 33 (1991), 575-596.

[W2] The Ricci flow on complete $\mathbb{R}^{2}$ (The limiting case of the porous medium equations as $m \rightarrow 0$ ), submitted.

\footnotetext{
Center for Math Analysis, Australian National University, Canberra, Act 2601

Australia

E-mail address: lang@gauss.anu.edu.au
}

Current address: Princeton University, Mathematics Department, Princeton, NJ 08544

E-mail address: lfwu@math.princeton.edu 